Mitteilungen der Österreichischen Geographischen Gesellschaft,

158. Jg. (Jahresband), Wien 2016, S. 13-36

\title{
Migration Und Integration MIGRATION AND INTEGRATION
}

\section{LEBEN IN ZWEI KULTUREN - TRANSNATIONALE IDENTITÄTEN indischer Migranten in Deutschland}

\author{
Carsten BuTsCH, Köln* \\ mit 1 Abb. im Text
}

\section{INHALT}

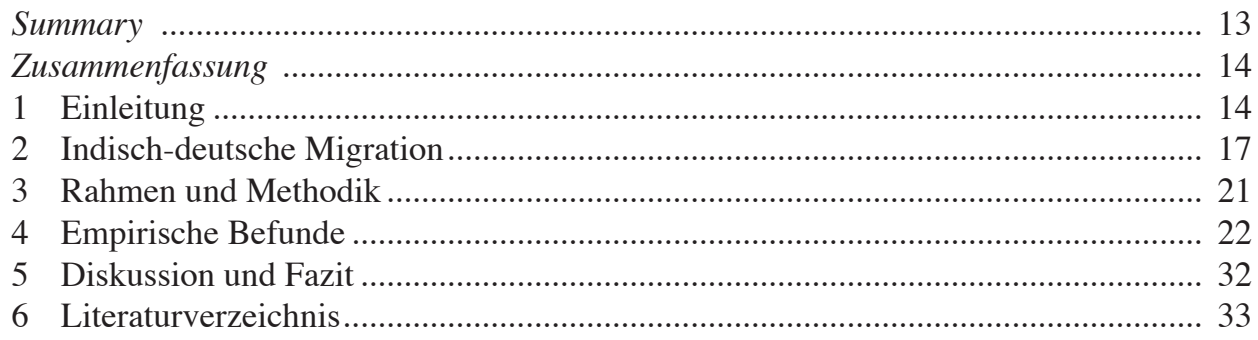

\section{Summary}

Living in two cultures - Transnational identities of Indian migrants in Germany

The question of migrant identities is a leitmotif of the research on transnationalism. This paper explores how Indian migrants in Germany describe their identities. Differing views will be portrayed: on the one hand positive self-perceptions, which are nurtured by living in two cultures; on the other hand the self-perceptions of transnational migrants, who perceive living transnationally as a burden. Further, it will be discussed how the relationship to the sending society develops with an increasing duration of stay in Germany. Also the cultural identity of different Indian migrant organisations in Germany will be described.

\footnotetext{
* Dr. Carsten Butsch, Diplom-Geograph, Geographisches Institut der Universität zu Köln, Otto-Fischer-Straße 4, Südbau, Zimmer 2.22, D-50923 Köln, Deutschland; E-Mail: butschc@uni-koeln.de,www.geographie.unikoeln.de
} 
Special interest is given to the analysis of transnational identities of second generation migrants. For this group the embedding in transnational familial and social networks poses a special challenge. The paper aims at substantiating the concept of "hybrid identities" by applying a differentiated analysis of the concrete case study.

Keywords: migration, transnationalism, identity, Germany, India

\section{Zusammenfassung}

Die Frage nach migrantischen Identitäten ist ein zentrales Motiv der Transnationalismusforschung. Im vorliegenden Beitrag wird erörtert, wie indische Migranten in Deutschland ihre Identität beschreiben. Dabei werden unterschiedliche Sichtweisen vorgestellt: $\ddot{A u ß e r s t ~ p o s i t i v e ~ S e l b s t b i l d e r, ~ d i e ~ d u r c h ~ d a s ~ L e b e n ~ i n ~ z w e i ~ K u l t u r e n ~ g e s p e i s t ~ w e r d e n, ~ s t e-~}$ hen dabei den Selbstbildern von Migranten gegenüber, die ihre transnationale Lebensweise als Belastung empfinden. Zudem wird dargestellt, wie sich das Verhältnis der Migranten zu ihrer Herkunftsgesellschaft entwickelt und auf welchem kulturellen Selbstverständnis unterschiedliche indische Migrantenorganisationen in Deutschland aufbauen.

Besonderes Augenmerk wird auf die Erfahrungen der zweiten Generation gelegt. Für diese Gruppe stellt die Einbindung in transnationale Familien- und Freundschaftsnetzwerke eine besondere Herausforderung dar. Der Artikel trägt durch die differenzierte Betrachtung zur inhaltlichen Anreicherung des Konzepts der „,hybriden Identitäten “ in der Transnationalismusforschung bei.

Schlagwörter: Migration, Transnationalismus, Identität, Deutschland, Indien

\section{Einleitung}

Like a turtle, you are always moving with the house on your back. You don't know where you're going to settle. Finally, you're looking for the sea. Somewhere, where you just go inside ... (eigenes Interview)

Die Frage nach migrantischen Identitäten ist ein zentrales Motiv der Transnationalismusforschung. Diese Forschungsrichtung befasst sich mit Phänomenen, die im Zuge der Globalisierung zugenommen haben und in früheren Theorien allenfalls als Marginalie erfasst wurden, nämlich mit der doppelten Einbindung von Migranten in ihre Herkunftsund Ankunftsgesellschaften (Glick Schiller, Basch \& Blanc-Szanton 1992). Durch diese neue Perspektive werden Grundannahmen bisheriger Migrationstheorien kritisch in Frage gestellt (Hillmann 2007, S. 78). Von besonderem Interesse sind die Beziehungen, die Migranten zwischen ihren Herkunftsorten und weiteren Orten schaffen. Diese sind vielfältiger geworden und haben im Globalisierungskontext eine neue Qualität erhalten (GoEKE 2007), während sich gleichzeitig die Migrationsmuster im globalen Maßstab verändert haben (Portes, Guarnizo \& Landolt 1999; Fassmann 2002). Durch neue Kommunikations- 
und Reisemöglichkeiten, vor allem aufgrund neuer technischer Möglichkeiten und gesunkener Kosten, wird das Leben an mehreren Orten logistisch einfacher, und werden die Kontakte zwischen Herkunfts- und Zielland enger und häufiger (PORTES 1996; FONER 1997; Portes, Guarnizo \& Landolt 1999; Vertovec 2001). Insbesondere die nahezu kostenlose internetbasierte Echtzeitkommunikation führt dazu, dass transnationale soziale Netzwerke effektiv in Globalisierungsprozesse eingebettet sind (VERTOVEC 2009). PRIES (2010) stellt fest, dass grenzüberschreitende Sozialbeziehungen aufgrund ihrer Häufigkeit und Dichte eine neue historische Qualität erreicht haben, und daher der Bruch zwischen Ausgewanderten und Daheimgebliebenen, der früher kennzeichnend war, nicht mehr in dem Maße auftritt.

In diesem Kontext stellt Identität ein zentrales Interessensgebiet dar (GoEKE 2007). Dabei wird erstens die Frage der „Hybridität“ von Identitäten und zweitens, darauf aufbauend, der Themenkomplex Inklusion-Integration-Assimilation diskutiert. Für VeRTOVEC (2001) bestehen zwei wesentliche Verknüpfungen zwischen Identität und Transnationalismus: (1) Gemeinsame Herkunft, Sprache etc. schaffen eine wahrgenommene gemeinsame Identität, welche die Grundlage für transnationale Netzwerke darstellt; (2) Identität wird in transnationalen Netzwerken an unterschiedlichen Orten verhandelt und konstruiert. In diesem Kontext werden hybride Identitäten oftmals als Kapital transnationaler Migranten beschrieben: „Within their complex web of social relations, transmigrants draw upon and create fluid and multiple identities grounded both in their society of origin and in the host societies“ (Glick Schiller, Basch \& Blanc-Szanton 1992, S. 11). Gleichzeitig erfordert die Schaffung ihrer hybriden Identitäten von den transnationalen Migranten die Fähigkeit, ihren Habitus in unterschiedlichen Kontexten zu wechseln (VerTOvec 2009). In ihrem Alltag verorten sie sich dabei irgendwo zwischen alleiniger Identifikation mit einer Gesellschaft und gleichwertiger Identifikation mit zwei Gesellschaften, wobei Letzteres einen Ausnahmefall darstellt (KRUMME 2004; LeVITT \& JAWORSKY 2007). Während hybride Identitäten von führenden Autoren der Transnationalismusforschung eher positiv beschrieben werden (z.B. VerTOVEC 2009), merken kritische Autoren an, dass die Chancen des Lebens in zwei Gesellschaften überbetont und die negativen Aspekte des Lebens zwischen zwei Kulturen ignoriert werden (z.B. BECKER 2002).

Identität berührt auch die Frage nach (doppelter) Staatsbürgerschaft (LEITNER \& EHRKAMP 2006). Für Staaten, die sich über Grenzen und (scheinbar) eindeutige Zugehörigkeit definieren, stellen hybride Identitäten, mit den gegebenenfalls auftretenden Loyalitätskonflikten, eine Herausforderung dar (VERTOVEC 2009). Dazu tragen auch die sich verändernden politischen Rahmenbedingungen bei, z.B. durch die verstärkte Einbindung ,der Diaspora“" in politische Prozesse (Vertovec 2001; Dickinson \& BAiley 2007). Dies hängt eng mit dem Diskurs über Integration und Assimilation zusammen. Dabei stehen sich in der Diskussion zwei unterschiedliche Standpunkte gegenüber: Während einige Autoren eine multiple Inklusion für undenkbar halten (ROBERTs et al. 1999; EsSER 2003) bzw. postulieren, dass Bindungen in die Heimat Inklusion in der Aufnahmegesellschaft verhindern (EHRKAMP 2005), sehen andere hierin eine neue, zeitgemäße Art der Assimilation (BrUBAKER 2001; Kivisto 2001; Schmiz 2011). Dies gilt besonders vor dem Hintergrund der (zunehmend) schwächer werdenden Kongruenz von Raum und Kultur, wodurch gesellschaftliche Zugehörigkeit nicht mehr ortsgebunden ist (GOEKE 2007). Allerdings wird - ebenfalls vor dem 
Hintergrund des Assimilationsdiskurses - infrage gestellt, wie dauerhaft transnationale Netzwerke sein können und insbesondere inwieweit transnationale Phänomene auch die sogenannte zweite Generation betreffen (GowrICHARN 2009).

Identität betrifft aber auch Veränderungen in den Herkunftsgesellschaften transnationaler Migranten. In transnationalen Netzwerken werden unter anderem Werte und Einstellungen verhandelt, wobei Migranten Veränderungen in ihren Herkunftsgesellschaften auslösen: „Social remittances are the ideas, behaviors, identities, and social capital that migrants export to their home communities. They may include ideas about democracy, health, gender, equality, human rights and community organization“ (LEVITT \& NyBERG-SøRENSEN 2004, S. 8). Die auf dem Transfer von Ideen beruhenden gesellschaftlichen Veränderungen sind ein Produkt der Kommunikationsleistung transnationaler Migranten. Verbesserte Kommunikationsmöglichkeiten und die gesunkenen Kosten hierfür tragen wesentlich dazu bei, dass social remittances an Bedeutung gewinnen. In den letzten beiden Dekaden hat sich das internationale Telefonaufkommen (gemessen in Anrufminuten) mehr als verzehnfacht (TeleGeography 2015, S. 2), während die Kosten für die Nutzer im gleichen Zeitraum um über $90 \%$ gefallen sind (ibid., S. 5). Zusätzlich haben sich in den letzten Jahren durch Smartphones und sogenannte messaging applications wie WhatsApp, Viber, WeChat oder Snapchat zusätzliche Kommunikationswege eröffnet, die ein konstantes In-Verbindung-Bleiben ermöglichen. Die Anzahl der aktiven Nutzer dieser Dienste hat sich von Dezember 2012 bis Juni 2015 von 500 Millionen auf 2,5 Milliarden erhöht (TeleGeOGRAPHY 2015, S. 7). Durch die hier angedeutete kommunikative Verflechtung werden neue Arten des Zusammenlebens, wie z.B. transnationale Elternschaft (CARLING et al. 2012), möglich, die den Transfer von Werten und Einstellungen, Verhalten, Sozialkapital etc. erleichtern.

Eng mit der multiplen Einbindung in soziale Strukturen verknüpft sind Finanztransfers (Rimessen) und das karitative Engagement von Migranten: „Monetary remittances have indeed become the most often cited, tangible evidence and measuring stick for the ties connecting migrants with their societies of origin." (GuARnizo 2003, S. 666) Rimessen erlangen in der globalisierten Welt zunehmende Bedeutung (LEVITT \& JAWORSKY 2007), weil sie entscheidend zu Veränderungen im Herkunftsland transnationaler Migranten beitragen. Gleichzeitig erfüllen Migranten spezifische Erwartungen im jeweiligen kulturellen Kontext ihrer Herkunftsgesellschaft. Dadurch sichern sie ihren sozialen Status im Herkunftsland und festigen ihre Netzwerke (BATNITZKy et al. 2012). Rimessen sind auf diese Weise eng mit der kulturellen Identität verknüpft - die in der Herkunftsgesellschaft geprägt wurde - und dienen der Versicherung der eigenen Identität.

Neben den individuellen Zahlungen, mit denen Migranten ihre Familien im Heimatland unterstützen, haben sich Arbeiten der Transnationalismusforschung vielfach mit sogenannten hometown associations befasst. Dies sind Migrantenorganisationen, die in engem Kontakt mit der Heimatregion der Migranten stehen und mit Spenden vor allem die dortige Infrastrukturentwicklung unterstützen (Portes 1996; Kivisto 2001; MutersbaUgh 2002; GuARnIzo 2003). In einigen Staaten hat diese ,private Entwicklungshilfe“ beträchtliche Ausmaße angenommen und die Abhängigkeit von der Diaspora sowie deren politischer Einfluss haben stark zugenommen (ITZIGSOHN 2000). Im internationalen Entwicklungsdiskurs werden Rimessen von verschiedenen Akteuren (Weltbank, Nichtregierungsorganisationen, Regierungen) zunehmend als wichtiger Beitrag zur „Entwicklung“ der „Heimat“ gesehen 
(FAIST 2008; Ho 2011), wodurch Migranten zunehmend als „Entwicklungsexperten“ instrumentalisiert werden (GEIGER \& STEINBRINK 2012). Allerdings entfalten diese spezifischen Investitionen nur punktuell Wirkung und es kann zu einem Rückzug von Staatlichkeit in der Planung kommen (VERTOVEC 2009). Dieses kollektive Engagement ist in doppelter Hinsicht mit der transnationalen Identität verbunden, da es einerseits in der identitären Verknüpfung mit der Heimatgesellschaft wurzelt und andererseits durch eine Gruppe gelebt wird, die in der Ankunftsgesellschaft durch die gemeinsame Herkunft konstituiert wird.

Hometown associations sind aber nur eine spezifische Ausprägung der sogenannten ethnic associations - eine Sammelbezeichnung für Vereine und Gesellschaften, deren Mitglieder sich auf eine, wie auch immer geartete, gemeinsame ethnische Identität berufen. Die Funktionen dieser Migrantenorganisationen können sehr vielfältig sein (FRIESEN 2008). Im Falle der hometown associations erfüllen sie die Funktion der Verknüpfung mit der Heimat. Andere Funktionen sind das gemeinsame Leben und Bewahren der Kultur der Herkunftsgesellschaft (insbesondere für die Kinder), gegenseitige Unterstützung (auch finanziell), das Leben religiöser Praktiken sowie das Bekanntmachen der eigenen Kultur in der Ankunftsgesellschaft (FrIESEN 2008). Die gemeinsame ethnische Identität, auf die sich die Mitglieder der Migrantenorganisationen berufen, ist dabei immer ein Aushandlungsprozess. Bei indischen Vereinen besteht eine wichtige Unterscheidung darin, ob es sich um Migrantenorganisationen handelt, die ein panindisches Verständnis zugrunde legen, oder um solche, die sich auf ein spezifisches Herkunftsgebiet innerhalb Indiens (und damit in aller Regel auf eine bestimmte Sprache und eine dominante Religion oder Richtung innerhalb des Hinduismus) berufen (FRIESEN 2008; GOWRICHARN 2009). Zwar gibt es gemeinsame identitätsstiftende Momente innerhalb der „,indischen Diaspora“, wie z.B. der Bezug zum indischen Unabhängigkeitskampf, der Stolz auf die wirtschaftliche Entwicklung der letzten Jahre oder sportliche Erfolge, vor allem beim Cricket, jedoch ist die Heterogenität deutlich größer als der häufig verwendete Begriff der ,indischen Diaspora“ impliziert (FRIESEN 2008).

Vor diesem Hintergrund wird in diesem Aufsatz der Frage nachgegangen, wie indische Migranten in Deutschland ihre transnationale Identität beschreiben und inwieweit sie in Wechselwirkung mit ihren transnationalen Praktiken stehen. Im Folgenden wird zunächst das deutsch-indische Migrationsgeschehen der letzten 70 Jahre skizziert. Daran anschließend werden Ziel und Methodik des Projekts beschrieben, in dessen Rahmen die verwendeten Daten erhoben wurden. Im empirischen Teil des Aufsatzes wird berichtet, wie indische Migranten ihre Identität darstellen und wie sich ihr Verhältnis zur Herkunfts- und zur Ankunftsgesellschaft entwickelt. Ein besonderes Augenmerk wird auf die transnationalen Identitäten der zweiten Generation gelegt. Der Aufsatz endet mit einer Diskussion der Ergebnisse und einem Fazit.

\section{Indisch-deutsche Migration}

Seit 1945 lassen sich insgesamt vier Phasen unterscheiden, in denen sich die indisch-deutsche Migration hinsichtlich ihres Volumens, der dominierenden Gruppe und 
damit der Migrationsmotivation jeweils grundlegend änderte. Diese Änderungen sind zum Teil durch Prozesse in Indien ausgelöst worden, zum Teil durch Prozesse in Deutschland. Als genereller Trend ist festzuhalten, dass die indisch-deutsche Migration insgesamt in den letzten 70 Jahren zugenommen und vor allem in den letzten 15 Jahren an Dynamik gewonnen hat.

Nach dem Zweiten Weltkrieg kamen zunächst Studenten nach Deutschland (Ost und West), angezogen durch die Reputation der deutschen Universitäten, insbesondere in den Fachbereichen Medizin und Ingenieurwissenschaften. Die meisten verließen Deutschland nach ihrer Ausbildung wieder, wenige blieben als hochqualifizierte, gut integrierte Arbeitskräfte, die oftmals deutsche Partner heirateten (GotTsCHLich 2012). Sie waren in Westdeutschland wesentlich an der Formierung der Deutsch-Indischen Gesellschaft (DIG) beteiligt, die sich als Gesellschaft zur Förderung der Völkerverständigung und -freundschaft begreift und die im Geist der deutschen Nachkriegsgesellschaft wurzelt. Ein wesentliches Anliegen ist die Vermittlung indischer Kultur in Deutschland, wobei eine inklusive Definition zugrunde gelegt wird (eigene Interviews). Da sich die Aktivitäten der einzelnen Zweiggesellschaften der DIG (jeweils lokal in 34 Städten organisiert) im Wesentlichen auf Deutschland fokussieren, können sie nicht als hometown associations im eigentlichen Sinne angesprochen werden, vielmehr dienen sie dem Zweck, indische Kultur in Deutschland bekannt zu machen.

Die zweite Phase der indisch-deutschen Migration ist durch den Zuzug indischer Gastarbeiterinnen im Gesundheitswesen gekennzeichnet. In den 1960er und 1970er Jahren kam eine große Zahl von Krankenschwestern und jungen Frauen, die in Deutschland als Krankenschwestern ausgebildet wurden (Goel 2013). Ein wichtiger Akteur in dieser Phase war die katholische Kirche, die dieses Netzwerk durch Anwerbung in Indien - vor allem im südindischen Bundesstaat Kerala - und die Bereitstellung von Ausbildungs- und Arbeitsplätzen in Deutschland ins Leben rief. Eine Besonderheit in dieser Phase ist die Dominanz der Frauen als wesentliche Akteure, vor allem, weil ein Teil dieser Migrantinnen während Heimaturlauben heiratete und ihre Männer als abhängige Familienmitglieder mitbrachten. Nach deutschem Ausländerrecht war diesen Männern für vier Jahre die Aufnahme einer eigenen Beschäftigung nicht gestattet. Dies brachte zahlreiche Konflikte mit sich, da die traditionelle Familienkonstellation der Herkunftsgesellschaft infrage gestellt wurde. Nach Goel (2013) stellt diese Situation einen wichtigen Grund dafür dar, dass die zur Untätigkeit verdammten Männer relativ schnell Vereine gründeten, die als Kerala-Kultur- oder Sportvereine Lebensinhalte und Abwechslung boten. Weitere wichtige Treffpunkte dieser Gruppierung waren Kirchen, in denen Messen auf Malayalam und nach dem Syro-malabarischen Ritus angeboten wurden. Aufgrund des einheitlichen kulturellen Hintergrunds (gemeinsame Herkunftsregion, gemeinsame Sprache, gemeinsame Religion) bildete sich ein starker Zusammenhalt unter den Migranten aus Kerala heraus, gleichzeitig grenzten sie sich dadurch von anderen indischen Migranten ab (Goel 2006; eigene Interviews). Mitte der 1970er Jahre befand man den Pflegenotstand für überwunden und in vielen Fällen erfolgte die nicht freiwillige Rückkehr der indischen Krankenschwestern und ihrer Familien nach Indien (Goel 2013), wobei insbesondere in Süddeutschland die Rückführung konsequenter umgesetzt wurde, weshalb diese Gruppe heute räumlich im Rhein-Ruhr-Gebiet konzentriert ist. Dieser Raum ist insgesamt ein 
Schwerpunkt in der räumlichen Verteilung indischer Staatsbürger in Deutschland. Weitere Schwerpunkte sind die westdeutschen Großstädte (München, Frankfurt am Main, Stuttgart und Hamburg mit jeweils über 3.000 indischen Staatsbürgern) sowie Berlin (4.448 indische Staatsbürger) $)^{1)}$ (vgl. Abb. 1).

Ab Beginn der 1980er Jahre, in der dritten Phase, dominierten geringqualifizierte Migranten aus dem Pandschab [Punjab] die Einwanderung nach Deutschland. Die überwiegend zur Religionsgemeinschaft der Sikhs gehörenden Zuwanderer kamen in aller Regel als Asylbewerber nach Deutschland. Auslöser dieser Migrationsbewegung war vordergründig die angespannte Situation im Pandschab, seit zu Beginn der 1980er Jahre die Khalistanbewegung an Bedeutung gewann und der Konflikt mit der Zentralregierung eskalierte (KHAN BANERJ \& SCHMIDT 2015). Während in Frage gestellt wird, ob tatsächlich politische Motive Ursache der Migration waren (und nicht vielmehr ökonomische Motive, vgl. Goel 2006; GotTschlich 2012; eigene Interviews), erfolgte in Deutschland eine starke Identifikation über die Religion und die Khalistanbewegung. Für diese Zuwanderergruppe stellen die Gebetshäuser der Sikhs (Gurdwaras) wichtige Treffpunkte dar. Auch diese Gruppe grenzt sich von anderen indischen Migranten ab. In der Folge kam es zu Konflikten in den bereits bestehenden Sikh-Gemeinden. Diese existierten oftmals lange vor Ankunft der Asylbewerber, die jedoch relativ schnell eine Majorität stellten und andere Vorstellungen von der Ausrichtung der Gemeinden hatten als die etablierten Gemeindemitglieder. Vor allem über das Verhältnis zur Khalistanbewegung gab es Konflikte, die zum Teil zur Abspaltung von Gemeinden führten (eigene Interviews).

In der aktuellen, vierten Phase hat die indisch-deutsche Migration stark zugenommen. Zudem wird die Migration abermals von neuen Gruppen dominiert, nämlich von Hochqualifizierten und Studierenden. Bei den Erstgenannten sind es vor allem Arbeitnehmer in der IT-Branche, die vermehrt seit den 1990er Jahren den Weg nach Europa suchen (KHADRIA 2014). In Deutschland wurden indische IT-Experten erstmals gezielt mit dem sogenannten „Greencard-Programm“ in den Jahren 2000 bis 2005 angeworben (KolB 2003). Die Änderungen des Zuwanderungsrechts 2005 und 2008 sowie die Bluecard-Regelung der Europäischen Union (EU), die 2012 eingeführt wurde (CHANDA \& MukHERJEE 2014; BAMF o.D. $\left.{ }^{2}\right)$, erleichtern insgesamt den Zuzug hochqualifizierter Arbeitskräfte, sodass sich die durch das Greencard-Programm angestoßene Migration verfestigt. Dazu trägt auch das zunehmende Engagement deutscher Firmen in Indien und indischer Unternehmen in Deutschland bei. Vor allem die Entsendung von Fachkräften ist ein wichtiger Migrationspfad. Geänderte Rahmenbedingungen betreffen auch die studentische Migration, die seit 2000 zugenommen hat: Während im Wintersemester 1999/2000 lediglich 539 indische Studenten ein Studium an einer deutschen Hochschule aufnahmen (Erstsemester), erreichte ihre Zahl im Wintersemester 2014/2015 bereits 4.051 (BAMF 2015, S. 7). Gründe für den starken Anstieg sind die Internationalisierung der deutschen Hochschulen, die gezielte Förderung von Austausch, unter anderem durch den Deutschen Akademischen

\footnotetext{
1) Angaben aus dem Ausländerzentralregister, auf Anfrage von den jeweiligen statistischen Landesämtern zur Verfügung gestellt

2) http://www.bamf.de/DE/Infothek/FragenAntworten/BlaueKarteEU/blaue-karte-eu-node.html (Zugriff Mai 2016)
} 


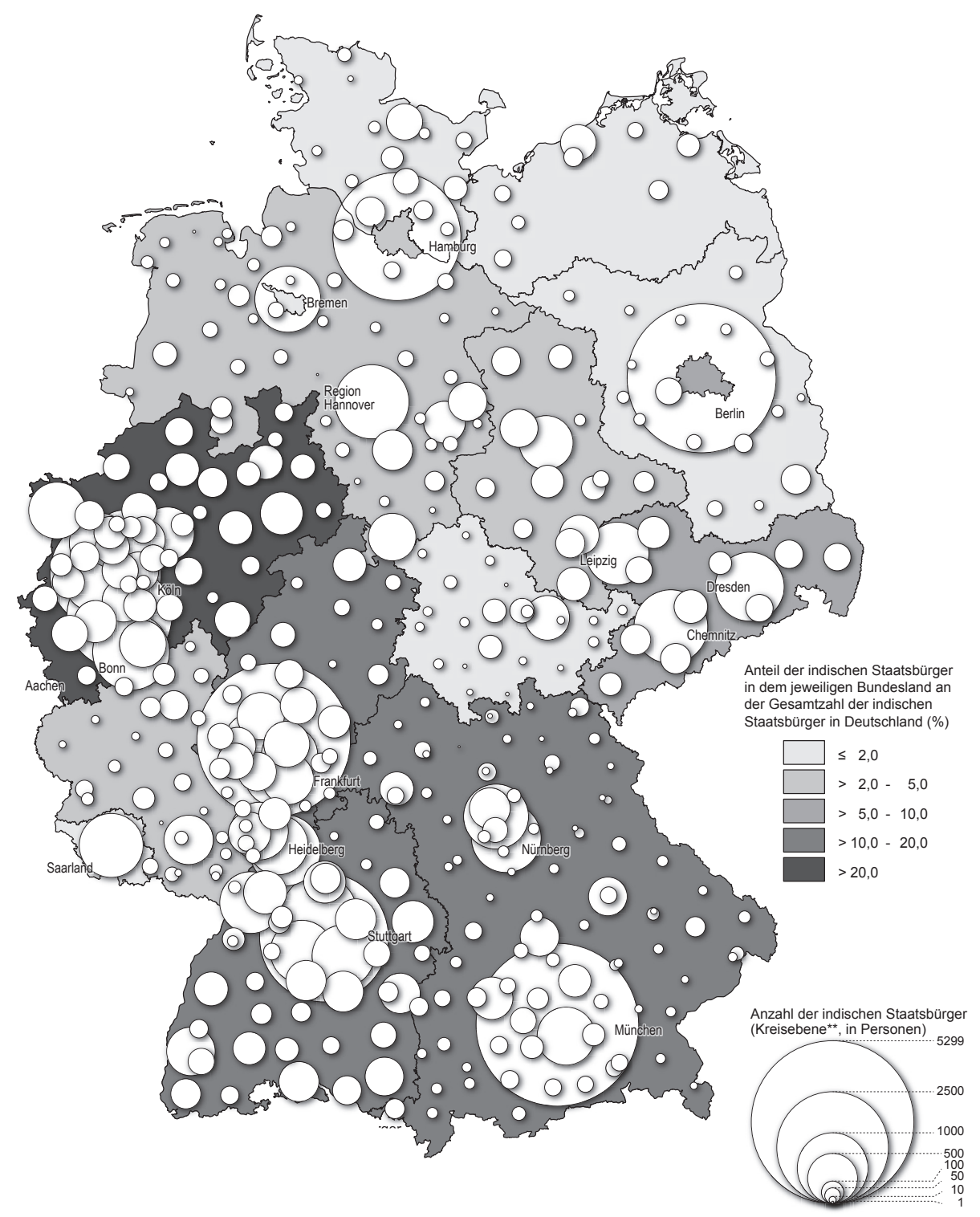

** gemeinsame Darstellung über Kreisgrenzen hinweg: Landkreis Kassel und Stadt Kassel, Kreis Aachen und Stadt Aachen, Stadt Cottbus und Landkreis Spree-Neiße (jeweils gemeinsame Ausländerbehörde).

Quellen: Ausländerzentralregister, Angaben der jeweiligen statistischen Landesämter auf Anfrage, sowie Statistisches Bundesamt (2016). Datenaufbereitung und Konzept: C. Butsch; Layout und Kartographie: R. SPOHNER

Abb. 1: Indische Staatsbürger in Deutschland, 31.12.2015 (Bayern: 31.12.2014) 
Austauschdienst, die gezielte Werbung der Hochschulen um indische Studenten (Mehrere Universitäten unterhalten eigene Verbindungsbüros in Indien, u.a. die Freie Universität Berlin, die Universität Heidelberg, die Rheinisch-Westfälische Technische Hochschule Aachen, die Technische Universität München, die Universität zu Köln und die Universität Göttingen.), Gebührenfreiheit und die hohe Reputation der Ausbildung an deutschen Universitäten, insbesondere in den Ingenieurwissenschaften.

Seit dem Jahr 2000 hat sich die Zahl der indischen Staatsangehörigen in Deutschland insgesamt mehr als verdoppelt, von 35.183 (31.12.2000) auf 86.324 (31.12.2015) (Statistisches Bundesamt 2005; Statistisches Bundesamt 2016). Der Mikrozensus 2014 schätzt die Zahl der Personen mit indischem Migrationshintergrund auf insgesamt 100.000 (Mitteilung vom Statistischen Bundesamt auf Anfrage). Vorliegende Statistiken deuten zudem auf enge transnationale Verflechtungen dieser Personengruppe hin. So ist die Aufenthaltsdauer der indischen Migranten insgesamt deutlich kürzer als die der restlichen ausländischen Bevölkerung (20\% kürzer als ein Jahr, 54\% kürzer als vier Jahre; ausländische Bevölkerung insgesamt: $10 \%$ bzw. 27\%; eigene Berechnung auf Grundlage von Statistisches Bundesamt 2015). Die Weltbank weist für das Jahr 2014 Rimessen aus Deutschland nach Indien in Höhe von 313 Millionen US Dollar ${ }^{3)}$ aus.

\section{Rahmen und Methodik}

Vor diesem Hintergrund wird in dem durch die Deutsche Forschungsgemeinschaft (DFG) geförderten Projekt „Transnationales Handeln indischer Migranten in Deutschland“ (THIMID) ${ }^{4)}$ der Frage nachgegangen, welche Verbindungen zwischen indischen Migranten in Deutschland, ihren Herkunftsorten in Indien und gegebenenfalls zu indischen Migranten in anderen Ländern bestehen. Die zentrale Forschungsfrage lautet: Warum und in welchem Maße sind in Deutschland lebende Personen mit indischem Migrationshintergrund in transnationale Netzwerke eingebunden und welche Veränderungsprozesse werden an unterschiedlichen Orten durch diese Netzwerke ausgelöst?

Zur Beantwortung dieser Frage wird in drei Forschungsphasen mit einem Multimethoden-Design gearbeitet (mixed method research [MMR]; AxINN \& PEARCE 2006; JoHNSON et al. 2007; BORK-HÜFFER 2012; BOSE 2012). Unterschiedliche Methoden werden genutzt, um die jeweiligen Schwächen einzelner Methoden auszugleichen, und es wird eine sequenzielle Integration verfolgt, um Forschungsfragen und Methodik innerhalb eines Forschungsprozesses weiterzuentwickeln. Dabei werden die Ergebnisse, die im frühen Stadium des Forschungsprozesses erzielt werden, genutzt, um Fragestellungen zu fokussieren und die Erhebungsinstrumente anderer Methoden im weiteren Verlauf des Forschungsprozesses auszugestalten. Die Verschränkung unterschiedlicher Methoden wird bei sequenzieller und nicht-sequenzieller Integration durch eine Triangulation (JICK 1979)

\footnotetext{
3) http://siteresources.worldbank.org/INTPROSPECTS/Resources/334934-1288990760745/Bilateral_ Remittance_Matrix_2014.xlsx (Zugriff Mai 2016)

4) DFG-Geschäftszeichen BU2747/1-1
} 
der Forschungsergebnisse in der Synthese (Phase vier) erreicht. Inhaltlich stellte die erste Phase des Projekts den Einstieg dar, in der mit Experten (Vertretern von Migrantenorganisationen, Botschaftspersonal, Konsulatspersonal) sowie mit Migranten semistrukturierte Interviews durchgeführt wurden. Themen waren dabei indisch-deutsche Migration (Motive, Entwicklung), die Vernetzung der indischen Gemeinde in Deutschland und die Muster transnationalen Handelns.

Eine weitere wichtige Methode war die teilnehmende Beobachtung. Treffen unterschiedlicher Migrantenorganisationen wurden besucht, um Interaktion zu beobachten und es wurden dabei zahlreiche unstrukturierte Gespräche geführt. Hierauf aufbauend wurde in der aktuell laufenden zweiten Phase eine quantitative Erhebung entwickelt und es wurden weitere, themenzentrierte semistrukturierte Tiefeninterviews geführt. In der anschließenden dritten Phase ist geplant, Migranten bei Reisen nach Indien zu begleiten und Interviews an ihren Herkunftsorten zu führen, um im Sinne der plurilokalen Feldforschung (multi-sited field studies - „follow the people“; MARcus 1995, S. 106) durch teilnehmende Beobachtung sowohl die Interaktion der Migranten an ihren Heimatorten zu untersuchen als auch mit Mitgliedern ihrer Netzwerke dort über Veränderungen, die durch die transnationale Einbettung hervorgerufen werden, zu sprechen.

Die folgende Darstellung der Migrationsgründe, der identitären Selbstbeschreibung und des damit in Zusammenhang stehenden transnationalen Handelns beruhen auf Erhebungen in der ersten und zweiten Projektphase. Wesentliche Quelle der Darstellung sind die semistrukturierten Interviews, von denen insgesamt 49 geführt wurden - davon elf mit Experten, 38 mit Migranten. Insgesamt wurden 22 Gespräche auf Englisch geführt, 27 auf Deutsch. 46 Interviews wurden mit Einverständnis der Gesprächspartner aufgezeichnet, anschließend transkribiert und mit MaxQDA ausgewertet. Die drei Interviews, in denen das Einverständnis zur Aufzeichnung nicht gegeben wurde, wurden direkt im Anschluss an das Gespräch aus dem Gedächtnis protokolliert und ebenfalls mit Max-QDA ausgewertet. Bei der Auswahl der Gesprächspartner wurde bewusst der Blick nicht allein auf transnationale Migranten im engeren Sinne - Personen, die fortlaufend transnational pendeln - gerichtet. Hiermit soll der systematische Fehler vermieden werden, der Transnationalismusforschern unterstellt wird, nämlich, dass aus wenigen, gezielt ausgewählten Fallbeispielen auf alle Personen mit Migrationshintergrund geschlossen wird (KING 2012, S. 144: ,sampling on the dependent variable“). Stattdessen ist es ein Anliegen, im Sinne der formulierten Forschungsfrage, die transnationale Einbettung, die migrantischen Identitäten und die transnationalen Praktiken indischer Migranten zu verstehen.

\section{Empirische Befunde}

Die identitären Selbstbeschreibungen der Interviewpartner der ersten und der zweiten Generation weisen eine sehr hohe Bandbreite auf. Äußerst positiven Beschreibungen stehen dabei durchaus verstörende Erfahrungen gegenüber. Gleichzeitig beschreiben die Probanden, wie sich ihre Identität nach der Migration durch das Leben in oder mit zwei Kulturen verändert. 
Tatsächlich beschreiben Probanden das Leben in zwei Kulturen und das daraus entstehende Selbstverständnis sehr positiv. So sagte eine Gesprächspartnerin, dass sie nach einigen Jahren in Deutschland für sich das Beste aus beiden Welten angenommen hat und dies als absolute Bereicherung empfindet. Eine andere Gesprächspartnerin fasst diese positive Kombination verschiedener Elemente folgendermaßen zusammen:

Befragte: „... so it was like, you have the fatherland and you also have the motherland. So it is like a nice combination."

Interviewer: „So what is the fatherland and what is the motherland? “

Befragte: „The fatherland is Germany, which is the fatherland, which you call the ,Vaterland" here and India is the motherland. So it was not like I gave up one mother to come to the other mother. No, it was a mother is there and a father comes also. It is a nice completion."

(Jyena, 42, lebt seit neun Jahren in Deutschland; Grund für die Migration war der Beruf ihres Mannes, der im mittleren Management eines deutschen Unternehmens tätig ist.)

Diese Beispiele stellen den positivsten Fall der in der Transnationalismusliteratur beschriebenen hybriden Identitäten dar. In Anlehnung an obenstehendes Zitat könnte dies als komplementäre Identität bezeichnet werden.

Eine andere Gesprächspartnerin beschreibt sich selbst als „Inderin mit deutschen Wurzeln“. Als Angehörige der zweiten Generation ist sie in Deutschland aufgewachsen, hat aber die indische Staatsbürgerschaft. Für sie war ein einschneidendes Erlebnis, dass ihr nach längerer Abwesenheit - sie hat in Indien studiert - zunächst kein Visum erteilt wurde, sodass nicht klar war, wann sie zu ihrer Familie und ihrem Partner (nach Deutschland) zurückkehren konnte. Bis zu diesem Zeitpunkt hat sie sich stark mit Deutschland identifiziert, durch dieses Ereignis ging jedoch das Zugehörigkeitsgefühl verloren. Daher hat sie sich für die oben genannte Selbstbeschreibung entschieden, die ihre Sozialisation in Deutschland, ihre Sozialisation als Kind indischer Migranten, ihr Leben in Indien und ihren rechtlichen Status zusammenfasst. Diesem identitären Selbstverständnis liegt eine kritisch-distanzierte Haltung zugrunde.

Neben diesen Sichtweisen, die eine gleichwertige Kombination aus beiden Kulturen für das eigene Selbstverständnis bemühen, gibt es andere Gesprächspartner, die sich stärker in einer Kultur beheimatet fühlen. Vor allem ältere Gesprächspartner mit deutschen Ehepartnerinnen beschreiben sich inzwischen selbst vor allem als Deutsche, ohne den Bezug zur indischen Kultur verloren zu haben.

Interviewer: „Aber das heißt, dass Sie auch, wenn Sie ihren Lebensmittelpunkt hier in Deutschland haben, schon auch eigentlich immer eine relativ enge Beziehung zu Indien hatten, wahrscheinlich auch über Air India? “

Befragter: „Genau richtig. Solange ich bei Air India war, musste ich ja ... ich war ja fast jeden Monat ein- oder zweimal in Indien wegen Konferenzen usw. Erstens das, zweitens mein Blut ist immer noch indisch. Das Blut können Sie nicht umtauschen. Das indische Blut ist da. [...]“ 
Interviewer: „Würden Sie sagen, Sie fühlen sich eher als Inder oder fühlen Sie sich eher als Deutscher? Sie haben gesagt, Ihr Blut ist indisch ..."

Befragter: „Ehrlich gesagt, ich fühle mich jetzt viel mehr als Deutscher als als Inder. In mehreren ... in manchen Sachen, meine Frau [eine Deutsche], sie ist eher Inderin als ich." (Lachen)

(Sriram, 83 Jahre alt, lebt seit 59 Jahren in Deutschland; ursprünglicher Migrationsgrund war die Aufnahme einer Beschäftigung bei der indischen Botschaft.)

Dabei beschreiben die Gesprächspartner dies zum Teil als aktive Anpassung, als willentliche Entscheidung. Dazu gehört auch, dass einige Befragte zum Beispiel für sie in Indien wichtigen Speisevorschriften aufgegeben haben. Personen brahmanischer Herkunft leben in aller Regel vegetarisch. Das hat insbesondere diejenigen, die in den 1960er, 1970er und 1980er Jahren kamen, bei ihrer Ankunft in Deutschland (mit den damals noch eher traditionellen Essensvorstellungen) zunächst vor große Schwierigkeiten gestellt. Als Anpassung an die Ankunftsgesellschaft wurden diese Gewohnheiten jedoch aufgegeben. Noch weitergehend berichtet der oben zitierte Gesprächspartner, dass er Katholik geworden ist, um sich in die Familie und das Gemeindeleben seiner Frau einzufügen. Ein anderer fasst seinen persönlichen Integrationsprozess mit den Worten zusammen: „Man muss aufhören Ausländer zu sein." Für ihn ist wichtig, dass seine Familie - trotz der Tatsache, dass er tatsächlich als einer der wenigen Befragten regelmäßig transnational pendelt - keine „Migrantenfamilie“ ist, sondern seine Kinder als Deutsche aufwachsen. Das Vermitteln „,deutscher Werte“ an die eigenen Kinder, um ihnen das Leben in Deutschland zu erleichtern, wurde auch von anderen Gesprächspartnern als wichtiges Erziehungsziel genannt.

Andere verorten sich stärker als Inder, auch wenn sie bereits seit langem in Deutschland leben:

„Indien ist meine Heimat. Also ich bin Fan von Indien, egal wie. Also ich bin hier auch glücklich, aber für mich ist Deutschland so etwas wie, wenn man berufstätig ist. Man hat eben einen Beruf, aber dann hat man ein Zuhause. Also für mich ist das so."

(Udipti, 62 Jahre alt, lebt seit 35 Jahren in Deutschland; ursprünglicher Migrationsgrund war ein Forschungsaufenthalt ihres Mannes.)

Andere beschreiben, dass die Differenzerfahrung in Deutschland sie dazu gebracht hat, sich stärker mit der indischen Kultur auseinanderzusetzen. Ein Interviewpartner sagte, dass er und seine Familie beispielsweise bestimmte Fastenzeiten viel strenger einhalten als die Familien seiner Geschwister in Indien. Eine andere Gesprächspartnerin sagt, dass sie in den ersten Jahren nach ihrer Migration nach Deutschland jedes indische Fest gefeiert und jedes Ritual ihrer Kindheit nachgeahmt hat, um die Beziehung zu ihrer Heimat zu erhalten. Nach dieser Phase setzte aber eine kritische Auseinandersetzung ein, die dazu geführt hat, dass sie inzwischen sehr reflektiert mit ihren indischen Traditionen umgeht.

Neben überwiegend positiven Bewertungen des Lebens in zwei Kulturen beschreiben andere den Aufenthalt in Deutschland negativ. Insbesondere zwei Gesprächspartnerinnen, 
die wegen des Berufs ihrer Ehemänner temporär in Deutschland leben, stellen die Migration als verstörend dar. Eine von ihnen wählt eine Geschichte aus dem Mahabarata, um den Zwischenzustand, in dem sie sich ihrer Ansicht nach befindet, zu illustrieren:

„There is a story in Mahabarat about a king called Trishanku. And when he dies, he wants to go to the heavens with his body. Now in Hindu concept this is not possible, because you burn the body and only your soul migrates to heavens. So anyway Trishanku prays to some god and this god grants him a boon saying ,You will ascent to the heavens in your body. 'So when he dies, he is actually going to the heavens with his body, but the god of heaven, Indra, he says: ,This is absolutely not possible, because I rule this place and I have never accepted a human with his body in heaven. He can't do that.' So he pushes him back. And this fellow who has granted him a boon is pushing to send him up - so Trishanku is hanging midway, he can't come down and he can't go up. So this is what happens to most people. They are Trishanku."

(Ganika, 41 Jahre, lebt seit neun Jahren in Deutschland; Grund für die Migration war der Beruf ihres Mannes, der in Deutschland für eine internationale Organisation tätig ist.)

Andere empfinden ihren Aufenthalt nicht per se negativ, fühlen sich aber von ihrer Umgebung zu Veränderungen genötigt, die sie selbst gar nicht wollen. So beschreibt ein Gesprächspartner, der ein mittelständisches Unternehmen leitet, dass er bewusst die Form der GmbH und einen deutschen Namen gewählt hat, weil er ein Unternehmen in Deutschland betreiben wollte. Seine Geschäftspartner haben ihn aber immer wieder gedrängt, Verbindungen nach Indien zu ermöglichen, weshalb er schließlich eine Dependance in Indien eröffnet hat und transnationale Dienstleistungen anbietet. In diesem Fall stimmte die Selbstwahrnehmung als ,deutscher Mittelständler“ mit der äußeren Zuschreibung als „IT-Inder“ nicht überein, was der Gesprächspartner nur widerwillig und aus ökonomischem Kalkül heraus akzeptiert. Ein anderer Gesprächspartner beschreibt eine ähnliche Erfahrung, jedoch in entgegengesetzter Richtung. Er hat den Eindruck, dass seine Karriere sich nur entwickelt, wenn er ,deutscher“ wird:

„India is my home, because it is India, which gave me this opportunity to develop my personality in such a way. And it is a kind of debt on me, you know? Means like here I do not mean to be very emotional or something, but that is my feeling. That is a debt on me, that India has prepared me such a way that I can do things which I am doing now. Okay, a very big contribution of that is from Germany as well. I can't ignore that. Definitely I have learned a lot, a lot, a lot in my last 8, 9 years. But my nursery is India. And I have been nursed in such a way I have, means such a way, which allowed me to reached to this point so. And you know - allow me to be a bit emotional (Lachen) - but here in my heart, from my heart I am Indian. I can't live without Indian food. I can't live without Indian music. I miss my family, my friends you know. So I think that India is my home somehow. [...]

So it is very ... there is a word „,bewildered“...I am at a point where I ask myself that 'OK, whether I am an Indian or German or what?' For my professional growth 
I need to be a German. That's clear to me. That's very clear to me, that I need to be a German if I need some growth in my career. But I can't leave my root."

(Ayush, 39 Jahre, lebt seit zehn Jahren in Deutschland; Grund für die Migration war eine Anstellung als Journalist in Deutschland.)

Dieser ,erzwungene“ Transnationalismus, der, wie gezeigt, auf unterschiedliche Weisen von außen an die Gesprächspartner herangetragen wird, führt ebenfalls zu einer identitären Verunsicherung. Dies kann, wie im ersten Beispiel, eine Ethnifizierung sein, also eine Reduzierung der Persönlichkeit auf ein Klischee oder aber der Zwang, bestimmte Verhaltensweisen abzulegen, um in der Ankunftsgesellschaft auf weniger Widerstand zu stoßen.

Ein interessantes Beispiel für die Differenzerfahrung zwischen Selbstwahrnehmung und Zuschreibung stellen auch die Erfahrungen dar, die zwei junge Sikhs in einem Interview beschrieben. Für sie ist der Sikhismus vor allem durch das Gebot der Nächstenliebe und des Respekts vor anderen geprägt. Aufgrund der äußeren Erscheinung (Männliche Sikhs tragen traditionell Turban und schneiden sich weder Bart noch Haare.) werden sie häufig als (vermeintlich radikale) Muslime angesprochen. Aufgrund dieser Differenzerfahrung und zahlreicher Diskriminierungserfahrungen (Ausschluss von Sportarten wegen des Turbans etc.) haben mehrere Sikhs der zweiten Generation, im Alter zwischen 20 und 30 Jahren, den Sikh-Verband Deutschland gegründet. Ihr Anliegen ist es, über Sikhismus aufzuklären und Vorurteile abzubauen.

Das Aufwachsen mit zwei Kulturen und das damit zum Teil einhergehende Fremdheitsgefühl oder die damit zum Teil einhergehende Erfahrung der eigenen Andersartigkeit ist für die zweite Generation eine bedeutende Erfahrung. Auffallend ist die intensive Auseinandersetzung der zweiten Generation mit der eigenen, transnationalen Identität. Diese wird von Gesprächspartnern als besonders intensiv während ihrer Zeit als Jugendliche und junge Erwachsene beschrieben. Für die Kinder der Migranten, die als Krankenschwestern oder deren Ehemänner nach Deutschland kamen, gab es eine Reihe von Seminaren, in denen sich die jungen Erwachsenen mit dem Aufwachsen zwischen zwei Kulturen beschäftigten. Eine Gesprächspartnerin beschreibt dies einerseits als ein Abarbeiten an den Wertvorstellungen der Eltern, andererseits als gemeinschaftlichen Versuch, eine eigene deutsch-indische Identität zu definieren. Ein (verspätetes) Produkt dieser Seminare ist das Buch InderKinder (Goel, Punnamparambil \& Punnamparambil-Wolf 2012), in dem Vertreter der zweiten Generation in unterschiedlichen Formaten über ihre Identität berichten.

Interessanterweise ist in der zweiten Generation eine ganze Reihe transnationaler Praktiken zu beobachten, die sich jedoch von denen der ersten Generation unterscheiden. Von allen interviewten Vertretern der zweiten Generation werden regelmäßige Besuche in Indien, vor allem bei den Großeltern erwähnt. Zusätzlich schildern mehrere Gesprächspartner einen engen Indienbezug im Rahmen der Ausbildung (Studium in Indien oder eines Fachs mit Indienbezug) oder im Rahmen des ausgeübten Berufs. Gleichzeitig wird das Verhältnis zur indischen Kultur auch problematisch beschrieben und die Orientierung in Indien fällt nicht leicht. So berichtet eine Gesprächspartnerin, deren Eltern während ihrer Kindheit nach Indien zurückkehren wollten, von dem „Kulturschock“, den sie erlebte: 
„Ich war acht und ja, bin ich dort hingekommenen, es war für mich ein totaler Kulturschock. Es war trocken, es war wüstig, es waren total viele Straßenköter ich hab' das gehasst, irgendwie. Es war aufregend bis zu dem Tag, als ich in die Schule kam. Das war eine Privatschule mit Karmeliterinnen-Ordensschwestern, super-streng. Klassen mit 65 Mädchen, also überfüllt sozusagen. English-medium ... ich konnte ja überhaupt kein Englisch und dann musste man ja Gujarati und Hindi dazu und Marathi zum Teil auch noch und ... eigentlich stand ich wie ein Ochs vorm Berg. [...] Und dann kam noch dazu dieser paramilitärische Führungsstil. Also man musste ja morgens die Nationalhymne singen und dann gab es ,march past‘, also man musste marschieren und dann so einen komischen Text aufsagen wie ,India is my country. All Indians are my brothers and sisters' und so weiter und so fort also (Lachen). Ich hab' das so auswendig gelernt und runtergebetet. Im Nachhinein hab ich mir gedacht: ,Ist das krass'. Also ich fand das wirklich wie so ein brain washing und so eine identitäre Nationalisierung meines Selbst. Also so ein Infiltrieren und ich konnte mir im Nachhinein vorstellen wie so Indoktrination funktioniert."

(Nakti, 45 Jahre, zweite Generation, lebte als Kind für ein Jahr in Indien und studierte in Indien.)

Da auch die Eltern der Gesprächspartnerin mit der Rückkehr nach Indien nicht zufrieden waren, beschloss die gesamte Familie wieder nach Deutschland zurückzukehren. Insgesamt rührt aus dieser Zeit ein zum Teil gebrochenes Verhältnis der Gesprächspartnerin zur indischen Gesellschaft. Auch aufgrund dieser Erfahrung stellt sie das - auch später - aus ihrer Sicht positiv verklärende Indienbild ihrer Eltern infrage.

Andere Befragte beschreiben ein Fremdheitsgefühl sowohl in Indien als auch in Deutschland, was insbesondere im jungen Erwachsenenalter zu einer intensiven Auseinandersetzung mit der eigenen Identität führt, wie ein Gesprächspartner berichtet:

„Und in Indien ist es so ... klar diese Identitätskrise, die wir auch eingangs besprochen haben, dass man sich in Phasen seines Lebens gefragt hat, Wohin gehöre ich?' Wenn ich in Indien bin, fühle ich mich fremd, wenn ich hier bin, bin ich eigentlich auch fremd ... das kann man aber irgendwie ... oder ich habe das für mich irgendwie geschafft, das zu überwinden, weil ich meine Funktion, meine Rolle anders verstehe heute. Aber als junger Mensch ist das halt etwas Anderes, weil man sich mit anderen Dingen identifiziert oder sich zwangsläufig mit anderen Dingen identifizieren muss. Nur jetzt, wo man sich gefestigter fühlt und seine Rolle für sich selber gefunden hat, sind diese Dinge nicht mehr so das Problem."

(Chanchul, 38 Jahre, zweite Generation)

Dieses Fremdheitsgefühl wird auch von anderen beschrieben. So sagt eine Gesprächspartnerin, dass sie auch zu ihrer Familie in Indien eine gewisse Distanz fühlt und sich in Indien insgesamt unsicher fühlt, weil sie die Muttersprache ihrer Eltern nicht spricht und sich nur auf Englisch verständigen kann. Gleichzeitig sprechen ihre Verwandten nur sehr schlecht Englisch. Eine Befragte berichtet von einem Erlebnis im Rahmen einer gemein- 
samen Indienreise mit einer Freundin, im Anschluss an einen Familienbesuch, das ihr Selbstverständnis berührt hat:

„... dann sind wir in einen Tempel reingegangenen, dann wurde ich da direkt am Eingang - also meine Freundin ist erst gar nicht auf die erste Stufe gekommen, so ungefähr, aber das war ein Vishnu-Tempel und das gibt es halt teilweise, dass da halt keine Nichthindus reindürfen - und dann wurde ich da wirklich rausgekegelt und ich meine, ich fand es halt richtig unverschämt. Aber das war dann für mich so der Wendepunkt, weil ich dachte: ,Okay, dann leckt mich am Arsch, dann bin ich jetzt auch Touristin.' Insofern war das so einerseits so ,Häh?' und anderseits aber auch befreiend ,Ja gut. Okay, dann halt nicht." “

(Sarala, 34 Jahre, zweite Generation)

Gleichzeitig beschreibt die Interviewpartnerin an einer anderen Stelle des Gesprächs, dass sie ein sehr enges Verhältnis zu Indien, ihren dortigen Verwandten und zu der dortigen Kultur hat. Auch in ihrem Lebensalltag spielt die Verbindung zur indischen Verwandtschaft eine große Rolle, weil sie mit ihnen in engem Kontakt steht und es eine große gegenseitige Anteilnahme am Leben der jeweils anderen gibt. Weiterhin sagt sie - im Gegensatz zum obenstehenden Zitat - dass sie es eigentlich genießt in Indien in der Masse unterzutauchen, weil dort alle so aussehen wie sie. In ähnlicher Weise formulierte eine andere Gesprächspartnerin der zweiten Generation, dass sie es in Indien angenehm empfindet, nicht „die Exotin“ zu sein.

Interessant ist die Beschreibung der transnationalen Identitäten der zweiten Generation durch die Eltern. Insbesondere diejenigen, die zum Studium nach Deutschland kamen und deutsche Frauen (Die Interviewpartner waren allesamt männlich.) geheiratet haben, legten in der Erziehung sehr viel Wert darauf, dass ihre (inzwischen erwachsenen) Kinder im deutschen Schulsystem und in der deutschen Gesellschaft integriert waren. Diese Gesprächspartner haben ihren Kindern beispielsweise auch nicht ihre eigene Muttersprache beigebracht, was einige von ihnen im Nachhinein bereuen. Sie beschreiben das Verhältnis ihrer Kinder zu Indien dementsprechend distanziert. So sagt einer, dass für seine Kinder Indien Ausland sei, ein anderer, dass seine Kinder Indien nicht richtig kennen, sondern nur „,aus Hotelzimmern heraus“. Ein weiterer beschreibt das Verhältnis seiner Kinder als „diffus“, während ein Interviewpartner von einer „heimatlosen Generation“ spricht.

Eine Gesprächspartnerin - die Familie kam vor zehn Jahren wegen des Berufs ihres Ehemanns nach Deutschland - erzählt von einem Bild, das ihr Sohn im Kunstunterricht gemalt hat. Darauf ist er selbst in einem Fluss zu sehen, dessen eines Ufer Indien darstellt, das andere Deutschland. Sie sagt, dass für ihre Kinder Indien ein sehr wichtiger Teil ihrer Identität ist und dass sie sich sehr stark mit ihrer indischen Herkunft identifizieren. Gleichzeitig sagt sie, dass sie sich nicht vorstellen kann, dass ihre Kinder einmal in Indien leben, für eine Rückkehr nach Indien ist es ihrer Ansicht nach nun zu spät: „We have crossed a point of no return.“

Andere Gesprächspartner mit Kindern im schulpflichtigen Alter können sich durchaus vorstellen, dass ihre Kinder in Indien leben. Um diese Option beizubehalten, wählen einige Eltern für ihre Kinder bewusst eine internationale Schule statt einer deutschen, 
da dies erlaubt, die Schullaufbahn an anderen Orten, unter anderem in Indien, nahtlos fortzusetzen. Ein wichtiger Aspekt scheint dabei zu sein, ob die Kinder in Indien oder in Deutschland geboren wurden und wie viel Zeit sie in Indien verbracht haben. Denn ein Gesprächspartner sagt, dass er sich eine Rückkehr nach Indien für seine ältere Tochter vorstellen könne, die auch plant in Indien zu studieren, während seine jüngere Tochter Schwierigkeiten mit Indien hat (trotz sehr häufiger Besuche in Indien) und zu Hause beispielsweise nur Deutsch spricht.

Vertreter der ersten Generation mit sehr unterschiedlichen Migrationsbiographien berichten übereinstimmend, dass für sie das Vertrautmachen ihrer Kinder mit der indischen Kultur die Motivation war, Migrantenorganisationen zu gründen. Durch die eigene Elternschaft hat ein Prozess eingesetzt, bei dem sich die Gesprächspartner noch einmal selbst stärker mit ihrer eigenen kulturellen Identität auseinandersetzten. Ihren Kindern möchten sie die Gelegenheit geben, indische Feste, die sie selbst auch aus ihrer Kindheit kennen, zu feiern. Denn auch für diejenigen, die ihren Lebensmittelpunkt in Deutschland sehen, bleiben indische Feste ein wichtiger Teil des Lebens, der nicht ersetzt werden kann, auch wenn eine gewisse Anpassung an das Leben in Deutschland erfolgt:

„Ich fühle mich natürlich, wenn ich drüben bin, ganz anders als wie hier. Das ist
jetzt schon fast 35 Jahre her, aber ich kann trotzdem zum Beispiel Weihnachtsfeier,
kann ich jetzt nicht so feiern, wie ein Deutscher feiern würde -oder Karneval. Also
wir tun einen Baum - für die Kinder wahrscheinlich mehr. Sieht gut aus und dann
haben wir Geschenke da und sonst was. Aber das man innerlich was macht, also
das muss im Knochen drin sein. Von Geburt an. Dann kann man es mehr feiern
oder machen halt, aber für mich ist immer noch Indien mein Heimatland."
(Utraj, 53 Jahre, lebt seit 37 Jahren in Deutschland, Migrationsgrund war der Beruf seines
Vaters.)

Um mit seinen Kindern auch die Feste seiner Kindheit zu feiern, engagiert er sich daher in einem Verein, der wichtige nordindische Feste wie Holi oder Diwalli feiert.

Migrantenorganisationen werden auch als ein Ort empfunden, an dem es möglich ist, sich leichter und intensiver auszutauschen. So sagt eine Gesprächspartnerin, die in einem Verein tätig ist, der ein panindisches Kulturverständnis zugrunde legt, dass es für sie einen großen Unterschied macht, ob sie sich mit Freunden indischer Herkunft oder Freunden anderer Herkunft unterhält. Bei den Indern braucht sie sich weniger zu erklären. Die Migrantenorganisationen, die sich an alle Inder richten, bemühen sich, ein breites indisches Kulturverständnis zugrunde zu legen. Zu diesen Vereinen gehören, neben der DIG Studentenorganisationen (z.B. die Heidelberg Indian Students Association) und weitere, lokale Kulturvereine, wie z.B. die Indian Association Bonn oder die Indian Association Hannover. Ein panindisches Kulturverständnis legen in aller Regel die Organisationen zugrunde, die sich an Hochqualifizierte richten. Demgegenüber sind sowohl die Vereine der Migranten aus Kerala, die im Wesentlichen auf die indischen Krankenschwestern und ihre Partner zurückgehen, auf diese Gruppe beschränkt. Dementsprechend wird häufig die Lokalsprache Malyalam gesprochen (im Gegensatz zu Englisch oder Hindi in den anderen Organisationen) und es werden vor allem keralesische Feste gefeiert. 
Ein Gesprächspartner, der lange in der DIG tätig war, beschreibt, wie es mehrfach vergeblich Versuche gab, die Gruppe aus Kerala in Aktivitäten einzubinden, z.B. indem das keralesische Onam-Fest in der DIG gefeiert wurde. Ähnlich verhält es sich mit den Sikhs, für die oftmals die Gurdwaras Zentrum ihres kulturellen Lebens sind. Da viele Sikhs ein gebrochenes Verhältnis zu Indien als Staat haben, ist das Verhältnis zu anderen indischen Migranten teilweise nicht einfach. Ein Befragter, selbst Sikh, sagt über diejenigen, die sich sehr stark über ihren Status als Flüchtlinge definieren, dass sie sich selbst „ein Ghetto“ geschaffen hätten, in dem sie nun leben.

Im Gegensatz zum teilweise problematischen Verhältnis einiger Sikhs bringen andere Indien vor allem Heimatgefühle entgegen. Dieses Motiv taucht in fast allen Gesprächen mit den Migranten der ersten Generation, zum Teil, wie dargestellt, auch mit der zweiten Generation auf. Eine Gesprächspartnerin sagt, die indische (sic!) Kultur ist stark und begleitet einen das ganze Leben hindurch, auch wenn man Indien verlässt. Andere sprechen vom Stolz, den sie empfinden, wenn sie an Indien und die Kultur ihrer Herkunftsregion denken.

Dieses emotionale Verhältnis wird häufig auch im Kontext der Staatsbürgerschaft thematisiert. Viele Gesprächspartner sagen, dass sie aus emotionalen Gründen ihre indische Staatsbürgerschaft behalten oder es als sehr schwer empfunden haben, ihre indische Staatsbürgerschaft gegen die deutsche zu tauschen. Dabei ist es seit 2006 für ehemalige Staatsbürger und deren Nachkommen bis zur vierten Generation möglich, sich als Overseas Citizen of India (OCI) zu registrieren. Dieser Status beinhaltet lebenslange visafreie Einreise nach Indien, das Recht sich uneingeschränkt wirtschaftlich zu betätigen und Bildungseinrichtungen zu nutzen. Wesentliche Einschränkungen sind lediglich das Verbot des Erwerbs landwirtschaftlicher Nutzflächen sowie die fehlende Möglichkeit zur politischen Partizipation. Beide Einschränkungen werden von den Gesprächspartnern aber als unerheblich angesprochen.

Ein weiteres, wiederkehrendes Motiv in den Interviews, vor allem mit der ersten Generation, ist das Gefühl der Entfremdung. Dieses Gefühl beruht einerseits auf der Veränderung der eigenen Identität durch das Leben in Deutschland, andererseits auf der Erfahrung, dass sich die indische Gesellschaft verändert. Insbesondere der Wertewandel in Indien wird dabei als Grund für ein zunehmendes Distanzempfinden genannt:

„Wir leben in einer Nostalgie, dass es vielleicht in Indien existiert, was damals existiert hat, in der Zeit, als wir damals Indien verlassen haben und wir wollen zurückkehren immer zu dieser Quelle. Aber es ist nicht mehr da. Die Urbanisierung findet statt, auch in Dörfern."

(Matthew, 78 Jahre, lebt seit 48 Jahren in Deutschland, Migrationsgrund war ein Volontariat bei einer deutschen Zeitung.)

Diese Veränderungen werden, wie im obenstehenden Zitat angedeutet, mit der wirtschaftlichen Öffnung Indiens und den damit verbundenen Transformations- und Globalisierungsprozessen in Verbindung gebracht. Dieser gesellschaftliche Wandel wird von mehreren Gesprächspartnern als verstörend empfunden. So beschreibt eine Interviewpartnerin ihr Unbehagen in Anbetracht der jüngeren Entwicklung, bringt aber auch ihre Hoffnung zum Ausdruck: 


\begin{abstract}
„Indien hat sich in letzter Zeit sehr schnell entwickelt und die Entwicklung ist sehr schnell passiert, und da kommen die Menschen manchmal gar nicht zurecht. Und deswegen merkt man manchmal, das ist zurzeit mehr Materialismus. Indien ist bekannt für Spiritualität und das ist so ein bisschen störend, dass da jetzt mehr Materialismus ist, das ist alles Impact von außen. Diese ganzen multinational firms, die [nach Indien] gegangen sind und die jungen Leute, die studiert haben und jetzt in solchen Firmen arbeiten, haben viel Geld. Und diese Gier und so. Das ist eine Phase jetzt und vielleicht in ein paar Jahren da wird es dort auch wieder gut werden, wie hier in Europa. Hier war das auch erstmal Entwicklung und dann kam irgendwo Wohlstand und dann gab es Zufriedenheit. Und das kommt dort auch bald." (Udipti, 62 Jahre alt, lebt seit 35 Jahren in Deutschland; ursprünglicher Migrationsgrund war ein Forschungsaufenthalt ihres Mannes.)
\end{abstract}

Auf der anderen Seite beschreiben die Gesprächspartner auch, wie sie sich durch das Leben in Deutschland selbst verändert haben. Ein Gesprächspartner sagt, dass er sich in seinem Verhalten inzwischen stark an ,deutschen' Normen wie zum Beispiel Pünktlichkeit orientiert. In seinem regelmäßigen Kontakt mit indischen Geschäftspartnern erlebt er oft ein anderes Verständnis von Pünktlichkeit, das er inzwischen als unhöflich und zeitraubend wahrnimmt. Veränderungen ihres Konsumverhaltens beobachtet eine andere Gesprächspartnerin an sich selbst. Den Kauf von gebrauchten Gegenständen auf einem Flohmarkt findet sie eine wohltuende Befreiung von dem in Indien weit verbreiteten Konsum, was bei ihrer Familie auf kein Verständnis stößt:

„When you go back to India, you see everybody is hoarding for something, which is about to happen. I don't know what. And you are shocked by the scale and the proportion of everything. And it takes a while to get into that traffic so to speak you know, because if you are not a part of the traffic. Then suddenly you'll become stingy and it is very difficult to navigate the two worlds, because on the one hand you have stopped consuming like that. You just buy what you immediately require. You also live frugally in other senses. Like for example, I go to the ,Flohmarkt'very often and I buy things, which I find interesting or useful. When I tell my mother for example I buy second hand shoes, she tells me: 'please take money from me and buy things for yourself. “ ”

(Ganika, 41 Jahre, lebt seit neun Jahren in Deutschland; Grund für die Migration war der Beruf ihres Mannes, der in Deutschland für eine internationale Organisation tätig ist.)

Die selbst beobachteten Veränderungen führen dazu, dass zum Teil die Möglichkeit, den Lebensmittelpunkt nach Indien zurückzuverlegen, infrage gestellt wird. Die Gesprächspartnerin, von der obenstehendes Zitat stammt, würde sehr gern dauerhaft nach Indien zurückkehren. Die Wintermonate hat sie in den letzten Jahren ohnehin dort verbracht und sie plant, sich dort eine selbstständige Existenz aufzubauen. Jedoch hat sie Angst, dass ihr Plan daran scheitert, dass sie sich inzwischen (trotz der regelmäßigen Aufenthalte) vom Leben in ihrer Herkunftsgesellschaft zu weit distanziert haben könnte; sie befürchtet Schwierigkeiten, sich wieder zu integrieren. 
Andere Probanden beschreiben ihre eigenen Versuche dauerhaft nach Indien zurückzukehren, die scheiterten. Die Gründe dafür sind vielfältig: Teilweise fiel es schwer, in Indien eine adäquate, dauerhafte Beschäftigung $\mathrm{zu}$ finden, teilweise hatten die Kinder Probleme mit dem Wechsel des Schulsystems, teilweise war es die Unzufriedenheit mit der Organisation des Berufsalltags. So berichtet beispielsweise ein Arzt, der nach seinem Studium in Deutschland nach Indien zurückkehren wollte, dass er sich nicht in die Strukturen des indischen Gesundheitswesens einfügen konnte. Deshalb ist er nach Deutschland zurückgekehrt und hat sein Berufsleben hier bestritten, gleichzeitig reiste er aber - inzwischen ist er an der Schwelle zum Rentenalter - mehrmals jährlich in seine Heimatstadt und engagiert sich dort in zahlreichen wohltätigen Projekten, zumeist im Gesundheitssektor. Das heißt, dass er zwar eine dauerhafte Rückkehr ausschloss, aber dennoch sehr enge Verbindungen nach Indien unterhält.

\section{Diskussion und Fazit}

Die dargestellten Ergebnisse deuten eine große Bandbreite transnationaler Identitäten an. Diese steht auch in Zusammenhang mit den unterschiedlichen Intensitäten transnationaler Praktiken der einzelnen Migranten. Nur wenige sind transnationale Migranten im engeren Sinne (ITZIGSOHN et al. 1999), aber alle interviewten Migranten sind in transnationale soziale Netzwerke eingebunden und schaffen Verbindungen zwischen ihren Herkunftsorten oder den Herkunftsorten ihrer Eltern und den Orten, an denen sie leben. Diese Einbindung in zwei unterschiedliche gesellschaftliche Kontexte beeinflusst das Selbstbild grundlegend. Die hybriden oder multiplen Identitäten, die in der Literatur als Kapital der Migranten beschrieben werden (Glick Schiller, Basch \& BlanC-Szanton 1992; LevitT \& JAWORSKY 2007; VerTOVEC 2009), stellen sich in diesem Fallbeispiel differenziert dar. In Bezug auf die Selbstbeschreibungen stehen an dem einen Ende des Spektrums die äußerst positiven Darstellungen, die als komplementäre Identitäten angesprochen wurden. Dabei kombinieren Migranten positive Aspekte aus dem Leben in beiden Gesellschaften. Am anderen Ende des Spektrums steht die Selbstbeschreibung derjenigen, die sich durch den Migrationsprozess entwurzelt fühlen, ohne eine neue Heimat gefunden zu haben. Sie befinden sich in einem „Trishanku-Zustand“ (in Anlehnung an die Geschichte aus dem Mahabarata). Zwischen diesen beiden Extremen finden sich zahlreiche Zwischenformen, von denen der „erzwungene“ Transnationalismus nochmals eine Sonderrolle einnimmt. Zwar gelingt es den Migranten in dieser Situation durch das Annehmen von Anforderungen, die von außen an sie gestellt werden, in ihrem Berufsleben Erfolge zu erzielen, jedoch erfolgt dies auf Kosten unerwünschter Veränderungen des eigenen Handelns.

Für die zweite Generation bedeutet die Einbindung in transnationale Netzwerke und das Leben in zwei Kulturen mit zum Teil sehr unterschiedlichen Wertesystemen oftmals eine identitäre Verunsicherung, insbesondre im jungen Erwachsenenalter. Auf die Frage nach dem Transnationalismus der zweiten Generation kann an dieser Stelle auch eine differenzierte Antwort gegeben werden: Für die eigene Identität ist die indische Herkunft in jedem Fall bedeutsam. Gleichwohl ist das Verhältnis zur Herkunftsgesellschaft der Eltern 
oft kritisch-distanziert, und die meisten bewegen sich dort zunächst nicht selbstverständlich allein - teils wegen Sprachbarrieren, teils, weil bestimmte Verhaltensmuster, Normen und Werte nicht bekannt sind. Gleichwohl bedingt die indische Herkunft eine intensive Auseinandersetzung mit der eigenen Identität, die zu unterschiedlichen transnationalen Praktiken führt, in mehreren Fällen auch zu transnationalen Verbindungen im Berufsleben.

Dargestellt wurde ebenfalls das unterschiedliche Selbstverständnis verschiedener indischer Migrantenorganisationen. Vor allem die Gruppen, die von Hochqualifizierten gegründet wurden, verstehen sich als panindische Organisationen, die Personen indischer Herkunft mit unterschiedlichem kulturellem Hintergrund (sprachlich, religiös) eine „Heimat" bieten möchten. Demgegenüber separieren sich die Kerala-Vereine und die Sikhs und fokussieren sich auf ihre eigene Gemeinschaft. Dabei geht es in allen Organisationen im Wesentlichen um das Leben indischer Kultur in Deutschland. Funktionen, wie sie für hometown associations beschrieben werden, nehmen diese Organisationen kaum wahr. Karitatives Engagement in Indien wird in den Interviews vor allem als persönliche Angelegenheit beschrieben.

Bezüglich des Verhältnisses zu Indien beschreiben viele Gesprächspartner eine mit der Dauer des Aufenthalts zunehmende Distanzierung. Insbesondere diejenigen, die mit deutschen Partnern verheiratet sind, verlegen ihren Lebensmittelpunkt nach Deutschland. Aber auch andere beschreiben Bruchstellen in ihrem Lebenslauf, an denen eine nahtlose Rückkehr nach Indien nicht mehr möglich ist, z.B. wenn die Kinder im deutschen Schulsystem integriert sind oder die eigene Integration im deutschen Arbeitsmarkt bereits erfolgt ist. Zusätzlich beschreiben Gesprächspartner eine Entfremdung in Bezug auf gesellschaftliche Werte (Urbanisierung, Konsumorientierung) an ihrem Herkunftsort. Dies deutet darauf hin, dass die vollständige Inklusion in zwei Gesellschaften dauerhaft nur schwer zu leisten ist.

Diese Fallstudie zeigt, dass die inzwischen etablierte transnationale Perspektive auf Migration wichtige Fragen in den Blick nimmt, die dazu beitragen, das Verhalten von Migranten zu verstehen. Gleichwohl bedarf es einer weiteren inhaltlichen Anreicherung des Konzepts, da einige Aspekte, z.B. die hier vertieft behandelte Frage nach transnationalen Identitäten, bisher oftmals einseitig oder oberflächlich behandelt wurden. Das Potenzial der transnationalen Perspektive für ein vertieftes Verständnis der Folgen von Migration in einer zunehmend global verflochtenen Welt ist bei Weitem noch nicht ausgereizt.

\section{Literaturverzeichnis}

Axinn W.G., Pearce L.D. (2006), Mixed method data collection strategies. Cambridge, Cambridge University Press.

Batnitzky A., McDowell L., Dyer S. (2012), Remittances and the Maintenance of Dual Social Worlds: The Transnational Working Lives of Migrants in Greater London. In: International Migration, 50, 4, S. 140-156.

Becker J. (2002), Hybride und andere Identitäten. Anmerkungen zur Transnationalismusdebatte. In: Becker J., Felgentreff C., Aschauer W. (Hrsg.), Reden über Räume. Region, Transfor- 
mation, Migration. Festsymposium zum 60. Geburtstag von Wilfried Heller (= Potsdamer Geographische Forschungen, 23), S. 7-20. Postdam, Universitätsverlag Potsdam.

Bork-HüFFER T. (2012), Migrants' Health Seeking Actions in Guangzhou, China. Individual Action, Structure and Agency: Linkages and Change. Stuttgart, Steiner Verlag.

Bose P.S. (2012), Mapping movements: interdisciplinary approaches to migration research. In: VARGAS-SiLVA C. (Hrsg.), Handbook of research methods in migration, S. 273-294. Cheltenham - Northampton, Edward Elgar Publishing.

Brubaker R. (2001), The Return of Assimilation? Changing Perspectives on Immigration and Its Sequels in France, Germany, and the United States. In: Ethnic and Racial Studies, 24, 4, S. 531-548.

Bundesamt Für Migration und Flüchtlinge (BAMF) (Hrsg.) (2015), Migrationsbericht 2013. Zentrale Ergebnisse. Nürnberg.

Bundesamt Für Migration und Flüchtlinge (BAMF) (Hrsg.) (o.D.), Blaue Karte EU. - http://www. bamf.de/DE/Infothek/FragenAntworten/BlaueKarteEU/blaue-karte-eu-node.html (Zugriff: Mai 2016).

Carling J., Menjívar C., Schmalzbauer L. (2012), Central themes in the study of transnational parenthood. In: Journal of Ethnic and Migration Studies, 38, 2, S. 191-217.

Chanda R., MukherJee D. (2014), Investment and Skilled Mobility Linkages between India and the EU. In: Tejada G., Bhattacharya U., Khadria B., Kuptsch C. (Hrsg.), Indian skilled migration and development: To Europe and back, S. 47-70. New Delhi, Springer Verlag.

Dickinson J., BAILEY A.J. (2007), (Re)membering diaspora: Uneven geographies of Indian dual citizenship. In: Political Geography, 26, S.757-774.

Ehrкamp P. (2005), Placing Identities: Transnational Practices and Local Attachments of Turkish Immigrants in Germany. In: Journal of Ethnic and Migration Studies, 31, 2, S. 345-364.

EsSER H. (2003), Ist das Konzept der Assimilation überholt? In: Geographische Revue, 5, 2, S. $5-22$.

FAIST T. (2008), Migrants as Transnational Development Agents: An Inquiry into the Newest Round of the Migration-Development Nexus. In: Population, Space and Place, 14, 1, S. 21-42.

Fassmann H. (2002), Transnationale Mobilität: Empirische Befunde und theoretische Überlegungen. In: Leviathan, 30, 3, S. 345-359.

Foner N. (1997), What's New About Transnationalism? New York Immigrants Today and at the Turn of the Century. In: Diaspora, 6, 3, S. 355-375.

FrIESEN W. (2008). The Evolution of 'Indian' Identity and Transnationalism in New Zealand. In: Australian Geographer, 39, 1, S. 45-61.

Geiger M., Steinbrink M. (2012), Migration und Entwicklung: Merging fields in Geography. In: Geiger M., Steinbrink M. (Hrsg.), Migration und Entwicklung: Geographische Perspektiven (= IMIS Beiträge, 42), S. 7-36. Osnabrück, Universität Osnabrück.

Glick Schiller N., Basch L., Blanc-Szanton C. (1992), Transnationalism: A New Analytic Framework for Understanding Migration. In: Annals of the New York Academy of Sciences, 645, S. 1-24.

Goeke P. (2007), Transnationale Migrationen. Post-jugoslawische Biografien in der Weltgesellschaft. Bielefeld, Transcript.

Goel U. (2006), Germany. In: LaL B. (Hrsg.), Encyclopedia of the Indian Diaspora. S. 358-360. Singapore, Editions Didier Millet.

Goel U. (2013), „Von unseren Familien finanziell unabhängig und weit weg von der Heimat“. Eine ethnographische Annäherung an Migration, Geschlecht und Familie. In: GeISEN T., StudER T., YILDIz E. (Hrsg.), Migration, Familie und soziale Lage - Beiträge zu Bildung, Gender und Care, S. 251-270. Wiesbaden, Springer. 
Goel U., Punnamparambil J., Punnamparambil-Wolf N. (2012), InderKinder. Über das Aufwachsen und Leben in Deutschland. Heidelberg, Draupadi Verlag.

Gotтschlich P. (2012), Carim-India Research Report 2012/03, German Case Study. San Domenico de Fiesole. - http://cadmus.eui.eu/handle/1814/20822 (Zugriff: Mai 2016).

GowrichaRn R. (2009), Changing forms of transnationalism. Ethnic and Racial Studies, 32, 9, S. $1619-1638$.

Guarnizo L.E. (2003), The Economics of Transnational Living. In: International Migration Review, 37, 3, S. 666-699.

Hillmann F. (2007), Migration als räumliche Definitionsmacht? Beiträge zu einer neuen Geographie der Migration in Europa (= Erdkundliches Wissen, 141). Stuttgart, Franz Steiner Verlag.

Ho E.L-E. (2011), ,Claiming' the diaspora: Elite mobility, sending state strategies and the spatialities of citizenship. In: Progress in Human Geography, 35, 6, S. 757-772.

Itzigsohn J., Cabral C.D., Hernandez Medina E., Vazquez O. (1999), Mapping Dominican transnationalism: narrow and broad transnational practices. In: Ethnic and Racial Studies, 22, 2, S. 316-339.

ItZIGSOHN J. (2000), Immigration and the boundaries of citizenship: the institutions of immigrants' political transnationalism. In: International Migration Review, 2000, S. 1126-1154.

JICK T.D. (1979), Mixing qualitative and quantitative methods: Triangulation in action. In: Administrative Science Quarterly, 24, 4, S. 602-611.

Johnson R.B., Onwuegbuzie A.J., Turner L.A. (2007), Toward a Definition of Mixed Methods Research. In: Journal of Mixed Methods Research, 1, 2, S. 112-133.

Khadria B. (2014), The Dichotomy of the Skilled and Unskilled Among Non-resident Indians and Persons of Indian Origin: Bane or Boon for Development in India? In: Tejada G., BhattaCharya U., Khadria B., Kuptsch C. (Hrsg.), Indian skilled migration and development: To Europe and back, S. 29-46. New Delhi, Springer Verlag.

Khan BanerJi B., Schmidt M. (2015), Einheit in Vielfalt? Politische Separationsbestrebungen in Indien am Beispiel Khalistan und Gorkhaland. In: Geographische Rundschau, 67, 1, S. $32-39$.

KING R. (2012), Geography and migration studies: retrospect and prospect. In: Population, Space and Place, 18, 2, S. 134-153.

Kivisto P. (2001), Theorizing transnational immigration: a critical review of current efforts. In: Ethnic and racial studies, 24, 4, S. 549-577.

Kolв H. (2003), „Green Card“ - eine qualitative und quantitative Analyse der Ergebnisse der Maßnahme. - http://www.bamf.de/SharedDocs/Anlagen/DE/Downloads/Infothek/Zuwanderungsrat/ exp-kolb-zuwanderungsrat.pdf?_blob=publicationFile (Zugriff Mai 2016)

Krumme H. (2004), Fortwährende Remigration: Das transnationale Pendeln türkischer Arbeitsmigrantinnen und Arbeitsmigranten im Ruhestand. In: Zeitschrift für Soziologie, 33, 2, S. $138-153$.

Leitner H., Ehrkamp P. (2006), Transnationalism and migrants' imaginings of citizenship. In: Environment and Planning, 38, 9, S. 1615-1632.

LevitT P., JaWorsky B.N. (2007), Transnational migration studies: Past developments and future trends. In: Annual Review of Sociology, 33, S. 129-156.

Levitt P., Nyberg-Sørensen N. (2004), The Transnational Turn in Migration Studies (= Global Migration Perspectives, 6). Geneva, Global Commission on International Migration (GCIM).

Marcus G.E. (1995), Ethnography in/of the world system: the emergence of multi-sited ethnography. In: Annual Review of Anthropology, 24, S. 95-117. 
Mutersbaugh T. (2002), Migration, common property, and communal labor: cultural politics and agency in a Mexican village. In: Political Geography, 21, 4, S. 473-494.

Portes A. (1996), Global Villagers: The Rise of Transnational Communities. In: The American Prospect. March-April, S. 74-77.

Portes A., Guarnizo L.E., LAndolt P. (1999), The study of transnationalism: pitfalls and promise of an emergent research field. In: Ethnic and Racial Studies, 22, 2, S. 217-237.

PRIES L. (2010), Transnationalisierung. Theorie und Empirie grenzüberschreitender Vergesellschaftung. Wiesbaden, VS Verlag für Sozialwissenschaften.

Roberts B.R., Frank R., LozAno-Ascencio F. (1999), Transnational migrant communities and Mexican migration to the US. In: Ethnic and Racial Studies, 22, 2, S. 238-266.

Schmiz A. (2011), Transnationalität als Ressource? Netzwerke vietnamesischer Migrantinnen und Migranten zwischen Berlin und Vietnam. Bielefeld, Transcript.

Statistisches Bundesamt (Hrsg.) (2005), Bevölkerung und Erwerbstätigkeit. Ausländische Bevölkerung sowie Einbürgerungen. Wiesbaden.

Statistisches Bundesamt (Hrsg.) (2015), Bevölkerung und Erwerbstätigkeit. Bevölkerung mit Migrationshintergrund - Ergebnisse des Mikrozensus. Wiesbaden.

Statistisches Bundesamt (Hrsg.) (2016), Bevölkerung und Erwerbstätigkeit. Ergebnisse des Ausländerzentralregisters. Wiesbaden.

TeleGeography (Hrsg.) (2015), TeleGeography Report Excecutive Summary. Washington, PriMetrica Inc.

Vertovec S. (2001), Transnationalism and identity. In: Journal of Ethnic and Migration Studies, 27, 4, S. 573-582.

VERTOVEC S. (2009), Transnationalism. London - New York, Routledge.

World BANK (Hrsg.) (2015) Bilateral Remittances Matrix 2014. - http://siteresources.worldbank. org/INTPROSPECTS/Resources/334934-1288990760745/Bilateral_Remittance_Matrix_2014.xlsx (Zugriff: Mai 2016). 